\title{
SCientific Oral Presentation Abstracts
}

7. https://www.cancer.org/research/cancer-facts-statistics/allcancer-facts-figures/ cancer-facts-figures-2018.html.

8. https://dermoscopedia.org/Melanoma.

9. https://dermoscopedia.org/Benign_Melanocytic_lesions.

\section{-10.5812/IRANJRADIOL. 99143}

\section{Automatic Assessment of Ki-67 Proliferation Index in Lymphoma}

Ramin Nateghi ${ }^{1,}$ *; Mansoor Fatehi ${ }^{2}$; Nasrin Shayanfar ${ }^{3}$; Fattane Pourakpour ${ }^{2}$; Wolfgang Pokieser ${ }^{4}$; Christophe Kornauth ${ }^{5}$

${ }^{1}$ Department of Electrical and Electronic Engineering, Shiraz University of Technology, Shiraz, Iran

${ }^{2}$ National Brain Mapping Lab, Tehran, Iran

${ }^{3}$ Department of Pathology, Iran University of Medical Sciences, Tehran, Iran

${ }^{4}$ Klinisches Institut für Pathologie, Medizinische Universität Wien, Vienna, Austria

${ }^{5}$ Labor für Klinische Zytologie, Institut für Pathologie des Wihelminenspials, Vienna, Austria

${ }^{*}$ Corresponding author: Department of Electrical and Electronic Engineering, Shiraz University of Technology, Shiraz, Iran. Email: r.nateghi.s@gmail.com

\section{Abstract}

Background:Haematopathological Ki-67 is used principally to measure the proliferation rate in the assessment and grading of malignancies. Ki-67 is based on a powerful staining method for distinguishing benign from malignant proliferation. The index uses a nuclear protein expression and it has been widely used to evaluate the proliferative activity of lymphoma. The clinical value of Ki-67 includes defining prognosis (among lymphomas), predicting drug response, and setting eligibility criteria for clinical trials. The Ki-67 score or index should be expressed as the percentage of positively stained cells among the total number of invasive cells in the area scored. With the Ki-67 marker, the proliferation fraction of low-grade follicular lymphomas (FLs) is usually less than $20 \%$ (as shown here) and that of high-grade FLs is greater than 30\% [1]. Manual Ki-67 proliferation assessment is a very time-consuming and operator-dependent task at the same time. Therefore, several studies have examined the use of image analysis software to measure faster the nuclear staining index of Ki-67 in lymphomas. A few studies have focused on the measurement of proliferation index in FLs and found that automated Ki-67 counts were similar to manual counts [2-3]. A major source of difference between automatic and manual Ki-67 scores is the scoring method that depends on the strategy of counting or the estimation and choice of the area to count.

Methods: In this research, an automatic unsupervised learning-based system was proposed for accurate and fast Ki-67 scoring in lymphoma. The proposed methods were designed to use image processing tools and detect robustly the positive and negative cells for Ki-67 antibody. The goal of the proposed method was to assess the proliferation index (percentage of Ki-67 positive lymphoma cells) to provide better treatment options for lymphoma patients. The proposed system consisted of the following sections: pre-processing, feature extraction, segmentation, and post-processing (Figure 1). To highlight specific histological structures of Ki-67 stained images such as positives cells (brown color ones), we performed pre-processing such as color transform from RGB space to brown-ratio space. For smoothing and filling the region of each cell on the image, the morphological filling was used. After the pre-processing section, color features, such as the mean of brown-ratio color space and blue channel of RGB image in a $3 \times 3$ block, were extracted from the image. In the next section of the proposed system, using the extracted color features, the image was segmented into three clusters by k-means clustering. After image segmentation, each of the positive and negative cells was post-processed. In the post-processing section, to split the merged cell, the morphological opening was used and finally, false segmented regions with small areas were removed.

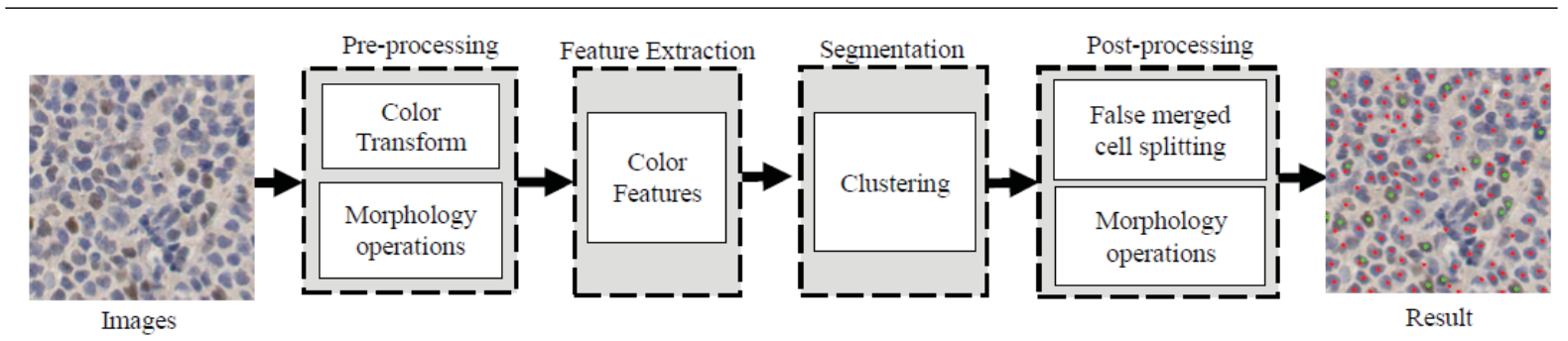

Figure 1. The proposed automatic Ki-67 assessment system 


\section{SCIENTIFIC ORAL Presentation AbStracts}

To evaluate experiments, we used five Ki-67 stained whole slide images of lymphoma from the Pathology Department of the Medical University of Vienna (AKH).
Results: Figure 2 shows the results of the proposed system for a sample region of the Ki-67 stained image.
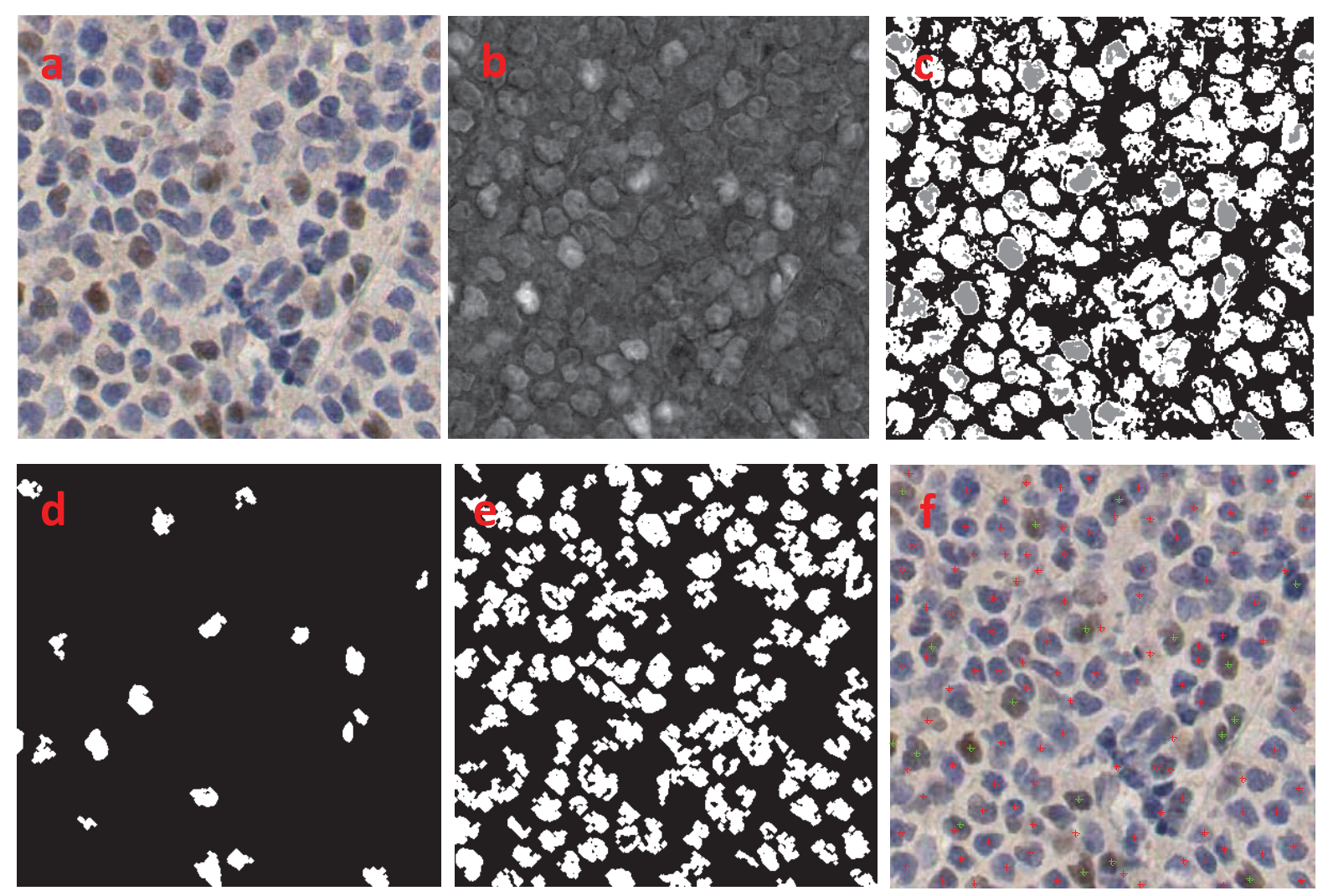

Figure 2. Results of the proposed system for a sample region of Ki-67 stained image: a, the sample region of a Ki-67 stained image, b, brown-ratio map, c, clustering, d, extracted positive cells, e, extracted negative cells, f, the final result with red dots representing negative cell centres and green dots representing positive cell centres

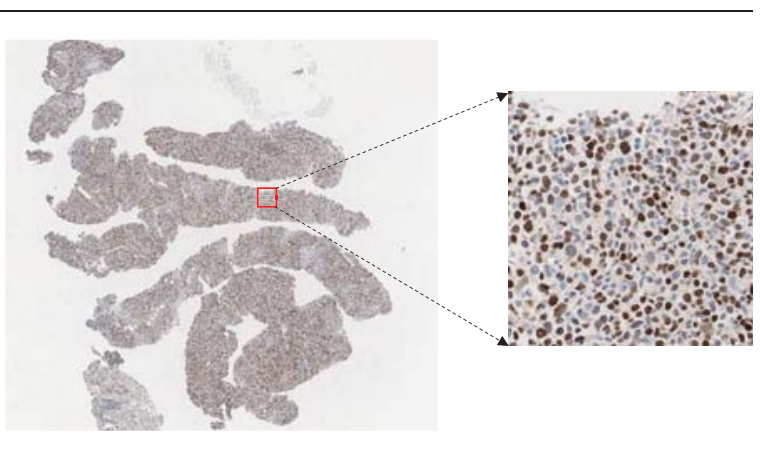

Figure 3. The sample whole slide image

The manually labeled positive and negative cells were considered as ground-truth. The ground-truth was compared with the automatically segmented cells obtained from the proposed system. The com- putational complexity of the proposed system was very low so that the average time needed for the assessment of a high-power field image with a resolution of $1239 \times 1239$ was 5.7 seconds using a workstation with a $2.50 \mathrm{GHz}$ Intel $®$ i7 - $2450 \mathrm{CPU}$ and a $16 \mathrm{~GB}$ memory. The efficiency of the proposed system was evaluated for estimating the Ki-67 index from Ki-67 stained whole slide images. A dataset was used containing five whole slide images. Figure 3 shows one of the whole slide images.

To compare the results, each slide was analyzed by an expert pathologist and the Ki-67 index was estimated manually. Table 1 shows the performance of the proposed system for the Ki-67 index estimation. The results confirmed the efficacy of the proposed system for Ki-67 index estimation from whole slide images.

Artificial Intelligence in Medical Imaging. 2019 November 


\section{SCientific Oral Presentation Abstracts}

Table 1. Performance of the proposed system for Ki-67 index estimation

\begin{tabular}{lcc}
\hline Slide & Manual & Automatic \\
\hline slide1.svs & $95 \%$ & $90.12 \%$ \\
\hline slide2.svs & $10 \%$ & $10.74 \%$ \\
\hline slide3.svs & $50 \sim 60 \%$ & $50.46 \%$ \\
\hline slide4.svs & $30 \sim 40 \%$ & $34.66 \%$ \\
\hline slide5.svs & $15 \%$ & $12.58 \%$ \\
\hline
\end{tabular}

Conclusion: The detailed experimental analysis reflected the promising results of Ki-67 scoring based on the proposed system.

Keywords: Lymphoma Cancer; Ki-67 Proliferation Index; Image Clustering; Immunohistochemistry

\section{References}

1. Naz, E., Mirza, T., Aziz, S., Ali, A., \& Danish, F, “Correlation of Ki 67 proliferative index with clinical and pathological features on tissue sections of non-Hodgkins lymphoma by immunostaining". JPMA-Journal of the Pakistan Medical Association, 748, 2011.

2. Martin AR, Weisenburger DD, Chan WC, "Prognostic value of cellular proliferation and histologic grade in follicular lymphoma", Blood, pp.3671-3678, 1995.

3. Zhang K, Prichard JW, Yoder S, "Utility of SKP2 and MIB-1 in grading follicular lymphoma using quantitative imaging analysis", Hum Pathol, pp. 878-882, 2007.

\section{-10.5812/IRANJRADIOL.99138}

\section{Automatic Fetal Biometry Evaluation in Ultrasound Images Using a Deep Learning- Based Approach}

Mostafa Ghelich Oghli ${ }^{1}$ *; Shakiba Moradi²; Reza Gerami ${ }^{3}$; Ali Shabanzadeh ${ }^{1}$
${ }^{1}$ Intelligent Imaging Technology Research Center, Med Fanavarn Plus Co., Karaj, Iran

${ }^{2}$ Sharif University of Technology, Tehran, Iran

${ }^{3}$ Army University of Medical Sciences, Tehran, Iran

${ }^{*}$ Corresponding author: Intelligent Imaging Technology Research Center, Med Fanavarn Plus Co., Karaj,Iran.Email: m.g31_mesu@yahoo.com

\begin{abstract}
Background:The 2D fetal ultrasound biometrics have been extensively used to establish (or confirm) the gestational age of the fetus, estimate its size and weight, and identify growth patterns and abnormalities. Typically, an ultrasound examination is routinely performed between 18 and 22 weeks of pregnancy to evaluate the growth of the fetus by measuring its head, abdomen, and femur. Automatic methods for fetal biometric measurements have been investigated recently to reduce intra-and inter-observer variability and create more accurate and reproducible measurements.

Objectives: In this paper, we proposed a deep learning-based approach to calculate fetal biometry parameters automatically.

Patients and Methods: The fetal biometry parameters came from the fetal head, abdomen, and femur evaluation. Head circumference (HC) and biparietal diameter (BPD) were related to the fetal head, whereas abdominal circumference (AC) was related to the fetal abdomen and femur length (FL) was related to the fetal femur. Figure 1 shows these parameters in ultrasound images.
\end{abstract}
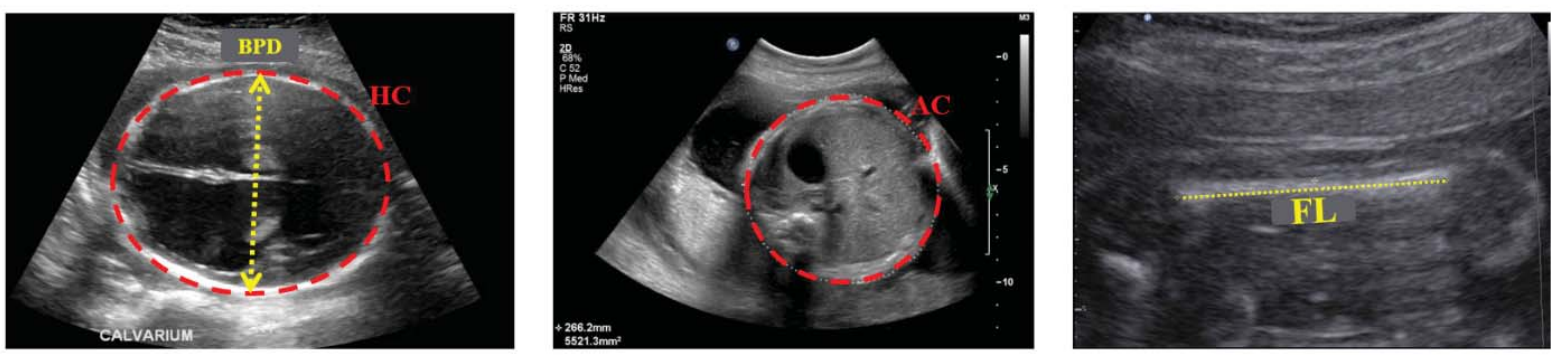

Figure 1. Parameters in ultrasound images

Our prepared dataset included three parts, as follows: (1) 1334 2D ultrasound images of the fetal head in the standard plane. This dataset was publically available from the automated measurement of fetal head circumference challenge. (2) 158 2D ultrasound images of the fetal abdomen in the standard plane. The dataset was gathered from $\mathrm{Al}-$ vand Medical Imaging Center, Tehran, Iran, by expert radiologists. (3) $3152 \mathrm{D}$ ultrasound images of the fetal femur in the standard plane. The dataset was gathered from two distinct centers: (i) Alvand Medical Imaging Center, Tehran, Iran, and (ii) Laleh Hospital, Tehran, Iran. We trained and evaluated a novel convolutional network for the segmentation of fetal head and abdomen. The proposed network, called MFP-Unet, was a combination of Unet and feature pyramid network (FPN). The network architecture is depicted in Figure 2. 